\title{
Relation between proton and neutron asymptotic normalization coefficients for light mirror nuclei and its relevance for nuclear astrophysics
}

\author{
N.K. Timofeyuk ${ }^{1, a}$, P. Descouvemont ${ }^{2}$, and R.C. Johnson ${ }^{1}$ \\ 1 Department of Physics, School of Electronics and Physical Sciences, University of Surrey, Guildford, Surrey GU2 7XH, UK \\ 2 Physique Nucléaire Théorique et Physique Mathématique, CP 229, Université Libre de Bruxelles, B-1050 Brussels, Belgium
}

Received: 20 June 2005 /

Published online: 15 March 2006 - (C) Società Italiana di Fisica / Springer-Verlag 2006

\begin{abstract}
It has been realised recently that charge symmetry of the nucleon-nucleon interaction leads to a certain relation between Asymptotic Normalization Coefficients (ANCs) in mirror-conjugated onenucleon overlap integrals. This relation can be approximated by a simple analytical formula that involves mirror neutron and proton separation energies, the core charge and the range of the strong nucleon-core interaction. We perform detailed microscopic multi-channel cluster model calculations and compare their predictions to the simple analytical formula as well as to calculations within a single-particle model in which mirror symmetry in potential wells and spectroscopic factors are assumed. The validity of the latter assumptions is verified on the basis of microscopic cluster model calculations. For mirror pairs in which one of the states is above the proton decay threshold, a link exists between the proton partial width and the ANC of the mirror neutron. This link is given by an approximate analytical formula similar to that for a bound-bound mirror pair. We compare predictions of this formula to the results of microscopic cluster model calculations. Mirror symmetry in ANCs can be used to predict cross sections for proton capture at stellar energies using neutron ANCs measured with stable or "less radioactive" beams.
\end{abstract}

PACS. 21.60.Gx Cluster models - 21.10.Jx Spectroscopic factors $-27.20 .+\mathrm{n} \quad 6 \leq A \leq 19-27.30 .+\mathrm{t}$ $20 \leq A \leq 38$

\section{Introduction}

Over the last 15 years, the nuclear physics community has shown a growing interest in Asymptotic Normalization Coefficients (ANCs). The study of these coefficients, both theoretically and experimentally, is mostly motivated by their application to nuclear astrophysics.

The one-nucleon ANC determines the magnitude of the large distance behaviour of the overlap integral between the bound state wave functions of nuclei $A$ and $A-1$. Such overlaps enter the amplitude for non-resonant nucleon capture reactions. If the capture occurs outside the nuclear interior, as often happens at very low stellar energies, then the overall normalization of its cross sections as well as of the astrophysical $S$-factors, is determined by the squared ANC [1]. Since the same ANCs play a crucial role in other peripheral processes such as transfer reactions, they can be measured in laboratories and used to predict non-resonant capture processes at low stellar energies [1].

\footnotetext{
${ }^{a}$ e-mail: n.timofeyuk@surrey.ac.uk
}

It has been suggested recently in ref. [2] that the ANCs of two mirror overlap integrals should be related if the charge symmetry of nucleon-nucleon $(N N)$ interactions is valid. It has been shown there that mirror ANCs can be linked by an approximate analytical expression which contains only nucleon separation energies, charges of the product nuclei and the range of the strong interaction between the last nucleon and the core. This link can be used to predict cross sections for non-resonant proton capture if mirror neutron ANCs are known. The latter can be determined using direct reactions in experiments with stable beams. Such experiments are less difficult and more accurate than ones involving radioactive beams which are necessary to determine the proton ANCs.

According to ref. [2], if one of the mirror nuclear states is a low-lying narrow proton resonance, and its mirror analog is particle-stable, then a link should exist between the width of the proton decaying state and the neutron ANC of its mirror analog. This link is given by an approximate analytical formula which is similar to that for the case of bound mirror pairs.

A proper understanding of the link between the width of a proton resonance and the neutron ANC of its mirror 
analog can be important for predicting the resonant proton capture rates for a particular class of resonant reactions at stellar energies. This class includes reactions that proceed via very narrow isolated states for which the proton width $\Gamma_{p}$ is either comparable to or much less than its $\gamma$-decay width $\Gamma_{\gamma}$. Such resonances can be found in the neutron-deficient region of the $s d$ and $p f$ shells (for example, some levels in ${ }^{25} \mathrm{Si},{ }^{27} \mathrm{P},{ }^{33} \mathrm{Ar},{ }^{36} \mathrm{~K}$ and $\left.{ }^{43,46} \mathrm{~V}\right)$ and their study is important for understanding nucleosynthesis in the $r p$ process. For the resonances mentioned above $\Gamma_{p}$ can be less than $1 \mathrm{eV}$. Direct measurements of such tiny widths using proton elastic scattering are impossible. Proton transfer reactions can be used instead. They provide spectroscopic factors which are combined with the single-particle widths to get the necessary partial proton widths. However, uncertainties in $\Gamma_{p}$ extracted through theoretical analysis of these reactions (for example, using the distorted-wave formalism) are about $50 \%$ [3]. These uncertainties arise due to problems in the theoretical analysis of stripping reactions to the continuum and the determination of the single-particle proton widths. The determination of proton widths based on the link to ANCs of their mirror particle-stable analogs can provide better accuracy since it avoids the uncertainties mentioned above.

In the present paper, we compare three different calculations of the ratio between mirror ANCs of bound mirror pairs and two calculations for the ratio between the proton widths and neutron ANCs of their mirror analogs. The first calculation uses the analytical formulae of ref. [2]. The second one (applied here only for bound mirror pairs) is based on the idea of mirror symmetry of single-particle potential wells and of spectroscopic factors. The third method uses the microscopic calculations within a multichannel cluster model. These three approaches are described in sect. 2, 3 and 4 . The comparison between them is given in sect. 5 and 6 and discussed in sect. 7 .

\section{Analytical formula}

\subsection{Bound mirror pairs}

The ANC $C_{l j}$ for the one-nucleon virtual decay $A \rightarrow(A-$ $1)+N$ is defined via the tail of the overlap integral $I_{l j}(r)$

$$
I_{l j}(r)=\left\langle\chi_{\frac{1}{2} \tau}\left[\left[Y_{l}(\hat{\boldsymbol{r}}) \otimes \chi_{\frac{1}{2}}\right]_{j} \otimes \Psi^{J_{A-1}}\right]_{J_{A}} \mid \Psi^{J_{A}}\right\rangle
$$

between the many-body wave functions $\Psi^{J_{A}}$ and $\Psi^{J_{A-1}}$ of nuclei $A$ and $A-1$. Here $l$ is the orbital momentum, $j$ is the total relative angular momentum between $A-1$ and $N, \tau$ is the isospin projection and $\chi_{\frac{1}{2} \tau}$ is the isospin wave function of nucleon $N$, and $r$ is the distance between $N$ and the center-of-mass of $A-1$. Asymptotically, this overlap behaves as

$$
\sqrt{A} I_{l j}(r) \approx C_{l j} \frac{W_{-\eta, l+1 / 2}(2 \kappa r)}{r}, \quad r \rightarrow \infty,
$$

where $\kappa=\left(2 \mu \epsilon / \hbar^{2}\right)^{1 / 2}, \epsilon$ is the one-nucleon separation energy, $\eta=Z_{A-1} Z_{N} e^{2} \mu / \hbar^{2} \kappa, \mu$ is the reduced mass for the $(A-1)+N$ system and $W$ is the Whittaker function.
For the $\left\langle\left.{ }_{N-1}^{A-1} Z \otimes n\right|_{N} ^{A} Z\right\rangle$ overlap, where the separated nucleon is a neutron, the ANC $C_{l j}$ can be represented by the matrix element $[4,5,6]$ :

$$
\begin{aligned}
& C_{l j}=-i^{-l} \frac{2 \mu \sqrt{A}}{\hbar^{2}} \times \\
& \left\langle\chi_{\frac{1}{2} \tau}\left[\left[j_{l}(i \kappa r) Y_{l}(\hat{\boldsymbol{r}}) \otimes \chi_{\frac{1}{2}}\right]_{j} \otimes \Psi^{J_{A-1}}\right]_{J_{A}}\left\|\hat{V}^{n u c l}\right\| \Psi^{J_{A}}\right\rangle,
\end{aligned}
$$

where $j_{l}(i \kappa r)$ is the spherical Bessel function,

$$
\hat{V}^{n u c l}=\sum_{i=1}^{A-1} V_{N N}\left(\left|\boldsymbol{r}_{i}-\boldsymbol{r}_{A}\right|\right),
$$

$V_{N N}$ is the strong two-body $N N$ potential and $\boldsymbol{r}_{i}$ is the radius-vector of the $i$-th nucleon.

For the mirror overlap $\left\langle{ }_{Z-1}^{A-1} N \otimes p \mid{ }_{Z}^{A} N\right\rangle$, where the separated nucleon is a proton, the expression for the ANC $C_{l j}$ can be obtained by considering the inhomogeneous coupled system of differential equations

$$
\begin{aligned}
& \left(\epsilon_{p}+\hat{T}_{l}^{r e l}+\sum_{n^{\prime} l^{\prime} j^{\prime}} V_{n l j, n^{\prime} l^{\prime} j^{\prime}}^{c o u l}(r)\right) I_{n^{\prime} l^{\prime} j^{\prime}}(r) \\
& =-\left\langle\chi_{\frac{1}{2} \tau}\left[\left[Y_{l}(\hat{\boldsymbol{r}}) \otimes \chi_{\frac{1}{2}}\right]_{j} \otimes \Psi^{J_{A-1}}\right]_{J_{A}}\left\|\hat{V}^{n u c l}\right\| \Psi^{J_{A}}\right\rangle,
\end{aligned}
$$

which is easily obtained from the simultaneous consideration of the Schrödinger equations for nuclei $A$ and $A-1$ and expanding the wave function of $A$ into complete set of eigenfunctions $\Psi_{n}^{J_{A-1}}$ of the Hamiltonian $H_{A-1}$ for the core $A-1$. In eq. (5) $V_{n l j, n^{\prime} l^{\prime} j^{\prime}}^{\text {coul }}(r)$ is the matrix element of the Coulomb interaction between the last proton and the protons of the core $A-1$ :

$$
\begin{aligned}
& V_{n l j, n^{\prime} l^{\prime} j^{\prime}}^{\text {coul }}(r)=\left\langle\left[\left[Y_{l}(\hat{\boldsymbol{r}}) \otimes \chi_{\frac{1}{2}}\right]_{j} \otimes \Psi_{n}^{J_{A-1}}\right]_{J_{A}}\right. \\
& \left.\times\left\|\sum_{i=1}^{A-1} \frac{e_{i} e_{A}}{r_{i A}}\right\|\left[\left[Y_{l^{\prime}}(\hat{\boldsymbol{r}}) \otimes \chi_{\frac{1}{2}}\right]_{j^{\prime}} \otimes \Psi_{n^{\prime}}^{J_{A-1}^{\prime}}\right]_{J_{A}}\right\rangle .
\end{aligned}
$$

Here $n$ denotes an excited state of $A-1, e_{i}$ is the charge of $i$-th nucleon and $r_{i A}=\left|\boldsymbol{r}_{i}-\boldsymbol{r}_{A}\right|$.

If non-diagonal Coulomb couplings in eq. (5) can be neglected and the diagonal Coulomb potential is replaced by a function $V_{m o d}^{\text {coul }}(r)$ which is a constant $V_{\text {mod }}^{\text {coul }}(r)=$ $\epsilon_{n}-\epsilon_{p}$ inside the Coulomb radius, and $(Z-1) e^{2} / r$ outside it, then the proton ANC is given by the same expression as eq. (3) but in which the Bessel function is replaced by the solution $\varphi_{l}^{\text {mod }}(r)$ of the Schrödinger equation with the potential $V_{\text {mod }}^{\text {coul }}(r)$. Since the main contribution to the ANC comes from internal nuclear region, we need to know $\varphi_{l}^{\text {mod }}(r)$ only at $r<R_{N}\left(R_{N}\right.$ is the radius of the nuclear interior). In this region it is given by the expression [2]

$$
\varphi_{l}^{m o d}(r)=\frac{F_{l}\left(i \kappa_{p} R_{N}\right)}{\kappa_{p} R_{N} j_{l}\left(i \kappa_{n} R_{N}\right)} j_{l}\left(i \kappa_{n} r\right), \quad r \leq R_{N}
$$

in which $i \kappa_{p}$ and $i \kappa_{n}$ are determined by the proton and neutron separation energies $\epsilon_{p}$ and $\epsilon_{n}$, and $F_{l}$ is the regular Coulomb wave function at imaginary momentum $i \kappa$. 
If the charge symmetry of $N N$ interactions is valid, then the wave functions of the mirror pairs ${ }_{Z}^{A} N-{ }_{N}^{A} Z$ and ${ }_{N-1}^{A-1} Z-{ }_{Z-1}^{A-1} N$ should be approximately the same in the nuclear interior and the ratio

$$
\mathcal{R}=\left(\frac{C_{p}}{C_{n}}\right)^{2}
$$

where $C_{p}$ and $C_{n}$ are proton and neutron ANCs for mirror nucleon decays, should be approximated by the square of the normalization coefficient in function $\varphi_{l}^{\bmod }(r)$ :

$$
\mathcal{R} \approx \mathcal{R}_{0} \equiv\left|\frac{F_{l}\left(i \kappa_{p} R_{N}\right)}{\kappa_{p} R_{N} j_{l}\left(i \kappa_{n} R_{N}\right)}\right|^{2} .
$$

\subsection{Bound-unbound mirror pairs}

It has been shown in ref. [7] that the partial width $\Gamma_{p}$ of a narrow proton resonance is related to the single-particle ANC $b_{p}$ of the Gamow function describing the proton motion in the resonance state times the spectroscopic factor $S_{p}: \Gamma_{p}=\left(\hbar^{2} \kappa_{p} / \mu\right) S_{p} b_{p}^{2}$. One can show that $S_{l}^{1 / 2} b_{p}$ can be represented by an integral containing the wave functions of nuclei $A$ and $A-1$ and the interaction potential between the proton and $A-1$ in the same way as for bound nuclear states. Assuming that the Gamow function in the internal region of the resonance is the same as the internal wave function of its mirror stable analog and repeating the reasoning of the previous section, we get for

$$
\mathcal{R}_{\Gamma}=\Gamma_{p} / C_{n}^{2},
$$

an approximate expression [2]

$$
\mathcal{R}_{\Gamma} \approx \mathcal{R}_{0}^{r e s}=\frac{\hbar^{2} \kappa_{p}}{\mu}\left|\frac{F_{l}\left(\kappa_{p} R_{N}\right)}{\kappa_{p} R_{N} j_{l}\left(i \kappa_{n} R_{N}\right)}\right|^{2} .
$$

In eq. (10) $C_{n}$ is the neutron ANC of the proton mirror bound analog. We would like to stress that the width $\Gamma_{p}$ entering eq. (11) is a residue in the $S$-matrix pole at the energy of the proton resonance and not the width for the cross sections of resonant reactions. However, for narrow resonances the difference between these two definitions of the width is small.

\section{Single-particle model}

According to the analytical formula, the ratio of mirror ANCs should depend only on nucleon separation energies and should be independent of the $N N$ potentials. We checked this property for the case of the two-body model. We considered a family of Woods-Saxon potentials that give some chosen neutron separation energy $\epsilon_{n}$, and some chosen proton separation energy $\epsilon_{p}$ when the Coulomb potential of a uniformly charged sphere was added. This was achieved by simultaneously varying both the depth and the radius of the Woods-Saxon potential at fixed diffusenesses. The actual numerical values of $\epsilon_{n}$ and $\epsilon_{p}$ were

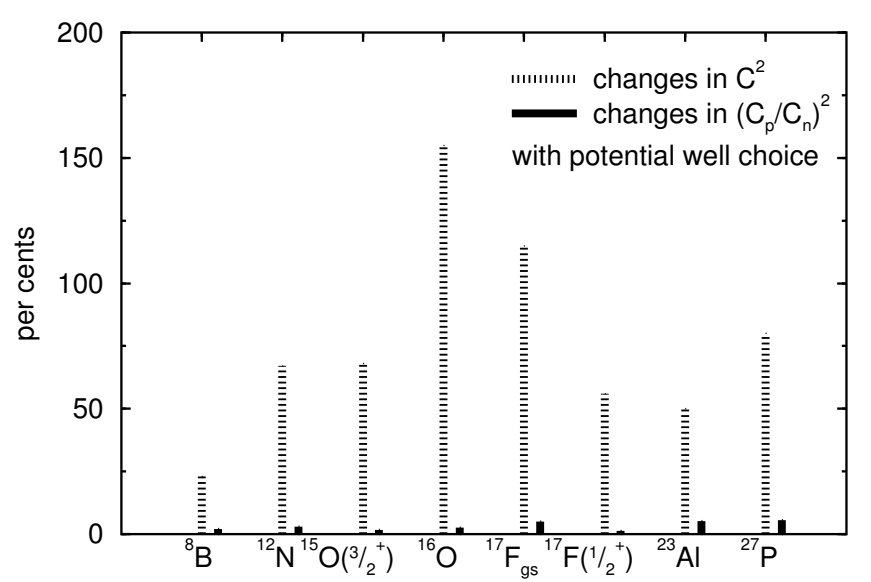

Fig. 1. Changes in ANCs squared and in ratio of proton to neutron ANC squared $\left(C_{p} / C_{n}\right)^{2}$ with choice of two-body nuclear potential well for a range of nuclei.

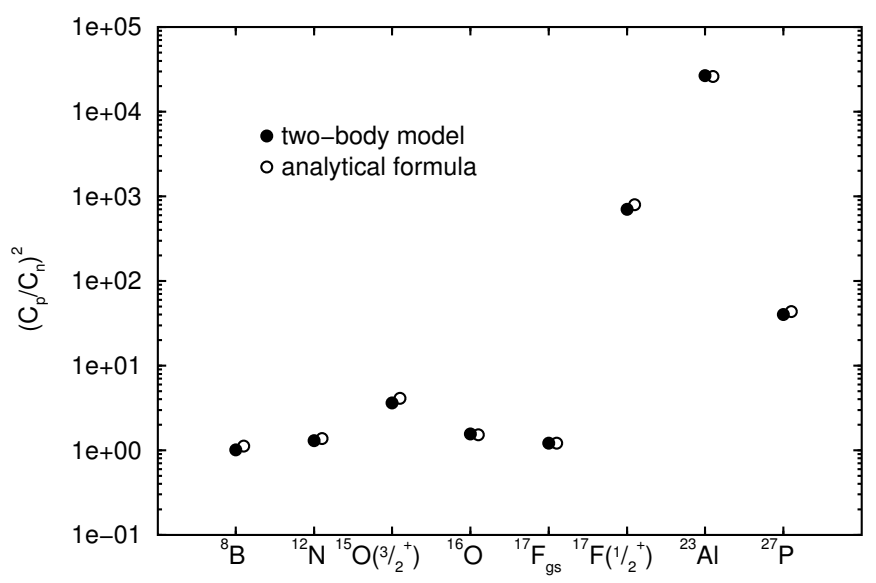

Fig. 2. Ratio of proton to neutron ANC squared $\left(C_{p} / C_{n}\right)^{2}$ calculated in the two-body potential model and using the analytical formula (9) for a range of nuclei.

the same as neutron and proton separation energies in the mirror pairs ${ }^{8} \mathrm{Li}^{8}{ }^{8} \mathrm{~B},{ }^{12} \mathrm{~B}_{-}{ }^{12} \mathrm{~N},{ }^{15} \mathrm{~N}\left(\frac{3}{2}^{+}\right)-{ }^{15} \mathrm{O}\left(\frac{3}{2}^{+}\right),{ }^{17} \mathrm{O}_{\text {g.s. }}{ }^{-}$ ${ }^{17} \mathrm{~F}_{\text {g.s. }},{ }^{17} \mathrm{O}\left(\frac{1}{2}^{+}\right)-{ }^{17} \mathrm{~F}\left(\frac{1}{2}^{+}\right),{ }^{23} \mathrm{Ne}^{-23} \mathrm{Al},{ }^{27} \mathrm{Mg}_{-}{ }^{27} \mathrm{P}$ and in the nucleus ${ }^{16} \mathrm{O}$.

For different potentials from the same family, the neutron and proton ANC values changed significantly but in such a way that their ratio was roughly the same. To illustrate this, we have presented in fig. 1 the changes in ANCs squared $C^{2}$ as thick vertical dashed lines and the changes in $\left(C_{p} / C_{n}\right)^{2}$ as vertical solid lines. While $C^{2}$ changes by 25 to $155 \%$, the changes in $\left(C_{p} / C_{n}\right)^{2}$ do not exceed $3 \%$.

The weak sensitivity of the ratio of mirror ANCs to the nuclear potentials suggests an alternative empirical way to determine this ratio. If we assume that mirror neutron and proton single-particle wells are exactly the same and that the spectroscopic factors $S_{p}$ and $S_{n}$ are equal for mirror pairs, then the ratio $\mathcal{R}$ can be approximated by the singleparticle ratio $\mathcal{R}_{\text {s.p. }}$

$$
\mathcal{R} \approx \mathcal{R}_{\text {s.p. }} \equiv\left(b_{p}^{c . s .} / b_{n}^{\text {c.s. }}\right)^{2},
$$


where the single-particle ANCs $b_{p}^{\text {c.s. }}$ and $b_{n}^{\text {c.s. }}$ are calculated numerically for exactly the same nuclear potential well. Unlike $\mathcal{R}_{0}, \mathcal{R}_{\text {s.p. }}$ takes into account the differences in internal wave functions of mirror nuclei due to the Coulomb interaction.

In fig. 2 we compare the ratio $\mathcal{R}_{\text {s.p. }}$ with the analytical estimate $\mathcal{R}_{0}$. One can see that $\mathcal{R}_{0}$ reproduces the general trend in $\mathcal{R}_{\text {s.p. }}$. well. The difference between them is about $2-6 \%$ for relatively large proton separation energies but can reach $10-20 \%$ for ${ }^{8} \mathrm{~B},{ }^{17} \mathrm{~F}\left(\frac{1}{2}^{+}\right)$and ${ }^{15} \mathrm{O}\left(\frac{3}{2}^{+}\right)$where this energy becomes very small.

\section{Microscopic cluster model}

To understand the validity of simple approximations (9), (11) and (12), their predictions should be compared to the numerical calculations using theoretical structure models. One of the models, the best adapted for ANC calculations, is a microscopic cluster model.

The multi-channel cluster wave function for a nucleus $A$ consisting of a core $A-1$ and a nucleon $N$ can be represented as follows:

$\Psi^{J_{A} M_{A}}=\sum_{l S J_{A-1} \omega} \mathcal{A}\left[\chi_{\frac{1}{2} \tau}\left[g_{\omega l S}^{J_{A-1}}(\boldsymbol{r}) \otimes\left[\Psi_{\omega}^{J_{A-1}} \otimes \chi_{\frac{1}{2}}\right]_{S}\right]_{J_{A} M_{A}}\right]$,

where $\mathcal{A}=A^{-\frac{1}{2}}\left(1-\sum_{i=1}^{A-1} P_{i, A}\right)$ and the operator $P_{i, A}$ permutes spatial and spin-isospin coordinates of the $i$ th and $A$-th nucleons. In this work, $\Psi_{\omega}^{J_{A-1}}$ is a wave function of nucleus $A-1$ with the angular momentum $J_{A-1}$ defined either in the translation-invariant harmonicoscillator shell model, or in a multicluster model. The quantum number $\omega$ labels states with the same angular momentum $J_{A-1}$ and $S$ is the channel spin. Transition from the $l S$ coupling scheme to the $l j$ coupling scheme can be done using standard techniques.

The relative wave function $g_{\omega l S}^{J_{A-1}}(\boldsymbol{r})=g_{\omega l S}^{J_{A-1}}(r) Y_{l m}(\hat{r})$ is determined using the R-matrix method. In this method, as explained in detail in ref. [8], the Bloch-Shrödinger equation is solved for the wave function $\Psi^{J_{A} M_{A}}$, which allows the correct asymptotic behaviour for the relative wave function $g_{\omega l S}^{J_{A-1}}$ to be obtained. For the states that are stable with respect to particle decay this behaviour is

$$
g_{\omega l S}^{J_{A-1}}(r) \approx C_{\omega l S}^{J_{A-1}} \frac{W_{-\eta, l+1 / 2}(2 \kappa r)}{r}
$$

and for particle-unstable states

$$
g_{\omega l S}^{J_{A-1}}(r) \approx A_{\omega} \frac{\delta_{\omega \nu} I_{\nu}\left(\kappa_{\nu} r\right)-U_{\omega \nu}^{J_{A-1}} O_{\nu}\left(\kappa_{\nu} r\right)}{\kappa_{\omega} v_{\nu}^{1 / 2}} .
$$

Here, $I_{\nu}$ and $O_{\nu}$ are the ingoing and outgoing Coulomb functions, $v_{\nu}$ is the velocity in the channel $\nu$ and $U$ is the collision matrix. The resonance width is determined by assuming a Breit-Wigner shape for the collision matrix near an isolated resonance. This width is the residue at the pole of the R-matrix and its ratio to the ANC squared of the mirror neutron can be compared to the approximation (11).

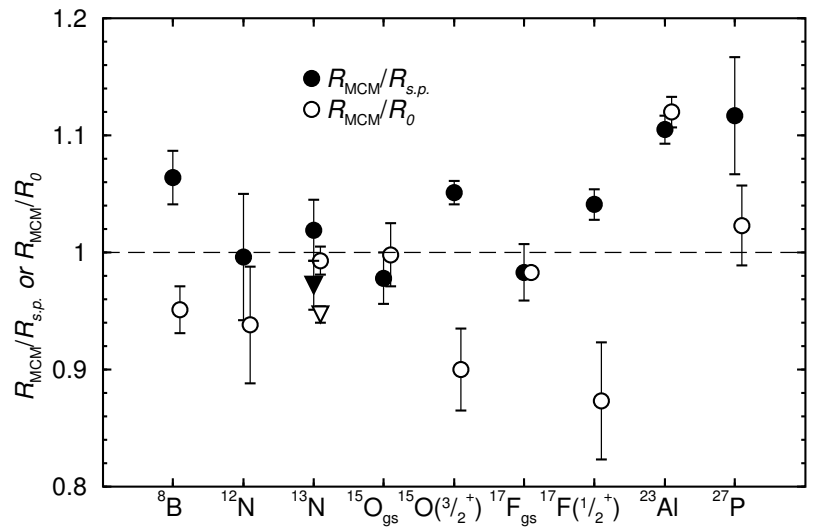

Fig. 3. Ratio $\mathcal{R}_{M C M} / \mathcal{R}_{0}$ and $\mathcal{R}_{M C M} / \mathcal{R}_{\text {s.p. }}$. For the ${ }^{13} \mathrm{C}_{-}{ }^{13} \mathrm{~N}$ mirror pair the circles represent the calculations within the four-cluster model and the downward triangles represent the calculations in the two-cluster model for various nuclei.

\section{Bound mirror pairs}

In this section we calculate mirror one-body overlap integrals for several $0 p$ and $s d$ shell nuclei within the multichannel cluster model and explore their properties such as ANCs, spectroscopic factors and single-particle ANCs. The residual nucleus is always taken in its ground state. We use the best adapted effective $N N$ interactions for such calculations, namely, the Volkov potential V2 [9] and the Minnesota (MN) potential [10]. The two-body spinorbit force [11] and the Coulomb interaction are also included. More details of the calculations can be found in ref. [12] and references therein.

Each of V2 and MN have one adjustable parameter that gives the strength of the odd $N N$ potentials $V_{11}$ and $V_{33}$. This parameter is usually fitted to reproduce the experimental separation energy for neutron or proton. Such a procedure is crucial for theoretical calculations of ANCs. However, in most cases the same choice of this parameter for mirror states does not reproduce both neutron and proton separation energies. Therefore, we use slightly different interactions in mirror nuclei to reproduce simultaneously the separations energies for neutrons and protons. This simulates charge symmetry breaking of the effective $N N$ interactions that should be a consequence of the charge symmetry breaking in realistic $N N$ interactions.

\subsection{Mirror symmetry in ANCs}

In this section we compare the ratio $\mathcal{R}_{M C M}$ of mirror ANCs squared obtained in the microscopic cluster model with two different approximations, $\mathcal{R}_{0}$ and $\mathcal{R}_{\text {s.p. }}$. The ratios $\mathcal{R}_{M C M}$ calculated with two different $N N$ potentials differed by no more than $4 \%$. So, we use for $\mathcal{R}_{M C M}$ the result averaged over two potentials V2 and MN. The calculated ratio $\mathcal{R}_{M C M} / \mathcal{R}_{0}$ and $\mathcal{R}_{M C M} / \mathcal{R}_{\text {s.p. }}$. are shown in fig. 3 for several mirror pairs. The error bars in this figure are due to averaging $\mathcal{R}_{M C M}$ over the two $N N$ potentials and because of uncertainties in $\mathcal{R}_{0}$ due to the choice of $R_{N}$ 
and the uncertainties in $\mathcal{R}_{\text {s.p. }}$ due to the residual dependence on the nucleon-core potential. Where two different values of the channel spin are possible, we take the sum of the squared ANCs in these channels for each of mirror nuclei and construct their ratio. In nuclear astrophysics the sum of the squared ANCs is often needed rather than their individual values in channels with different spin.

For the mirror pair ${ }^{13} \mathrm{C}-{ }^{13} \mathrm{~N}$, two models were used: a two-cluster model (downward triangles) and a four cluster model (circles). The slightly different ratios $\mathcal{R}_{M C M}$ obtained in these models are due to the different amount of charge symmetry breaking required in each of these models.

According to fig. 3, the deviation of $\mathcal{R}_{M C M}$ from the analytical value $\mathcal{R}_{0}$ does not exceed $7 \%$ for most cases except for the two $s$-wave mirror states with one node ${ }^{15} \mathrm{~N}\left(\frac{3}{2}^{+}\right)-{ }^{15} \mathrm{O}\left(\frac{3}{2}^{+}\right)$and ${ }^{17} \mathrm{O}\left(\frac{1}{2}^{+}\right)-{ }^{17} \mathrm{~F}\left(\frac{1}{2}^{+}\right)$and for the mirror pair ${ }^{23} \mathrm{Ne}^{23} \mathrm{Al}$. The deviations in these cases are $10 \%$, $13 \%$ and $12 \%$ respectively. In most cases $\mathcal{R}_{M C M}$ is smaller than $\mathcal{R}_{0}$ but larger than $\mathcal{R}_{\text {s.p. }}$. The deviation of $\mathcal{R}_{\text {s.p. }}$ from $\mathcal{R}_{M C M}$ is not more than $6 \%$ except for the ${ }^{23} \mathrm{Ne}^{23} \mathrm{Al}$ and ${ }^{27} \mathrm{Mg}_{-}{ }^{27} \mathrm{P}$ mirror pairs where these deviations are $10 \%$ and $12 \%$, respectively.

We have found that the average $\mathcal{R}_{a v}$ of two different approximations $\mathcal{R}_{0}$ and $\mathcal{R}_{\text {g.s. }}$ is in reasonably good agreement with $\mathcal{R}_{M C M}$. For all cases except ${ }^{23} \mathrm{Ne}^{23} \mathrm{Al}$ the difference between $\mathcal{R}_{a v}$ and $\mathcal{R}_{M C M}$ does not exceed $6 \%$. Therefore, in the absence of detailed microscopic calculations $\mathcal{R}_{a v}$ can be a good choice for using mirror symmetry in ANCs to predict proton ANCs from mirror neutron ones and vice versa.

The $10-12 \%$ difference between $\mathcal{R}_{0}$ (or $\mathcal{R}_{\text {s.p. }}$ ) and $\mathcal{R}_{M C M}$ for the mirror pair ${ }^{23} \mathrm{Ne}^{23} \mathrm{Al}$ arises due to strong core excitation effects. This deviation occurs in multichannel calculations which include many excited states in the ${ }^{22} \mathrm{Ne}$ and ${ }^{22} \mathrm{Mg}$ cores. When all the core excitations are removed, the calculated value of $\mathcal{R}_{M C M}$ decreases and agrees with $\mathcal{R}_{\text {s.p. }}$ and $\mathcal{R}_{0}$ within $2 \%$ [12].

\subsection{Mirror symmetry in spectroscopic factors}

The spectroscopic factor $S_{l j}$ is defined as

$$
S_{l j}=A \int_{0}^{\infty} \mathrm{d} r r^{2}\left(I_{l j}(r)\right)^{2},
$$

and we obtain them by numerical integration of the overlap integrals squared, calculated in the MCM. We use slightly different odd $N N$ interactions in each mirror state in order to reproduce the experimental separation energies both for neutrons and protons. However, these interactions do not differ much and, therefore, the difference in mirror wave functions in the nuclear interior should arise because of the charge symmetry breaking due to the Coulomb interactions. Since the latter are smaller then strong interactions and because the main contribution to the spectroscopic factor comes from nuclear interior, one expects that the spectroscopic factors in mirror states to be almost equal.

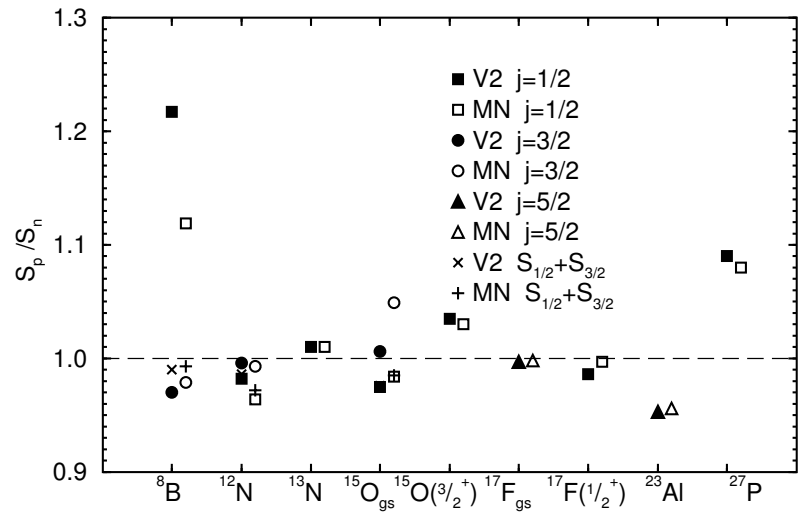

Fig. 4. Ratio of proton to neutron spectroscopic factors for various nuclei.

The ratio $S_{p} / S_{n}$, where $S_{p}$ is the proton spectroscopic factor and $S_{n}$ is the spectroscopic factors for its mirror analog, is shown in fig. 4 both for the V2 and the MN potentials. In this figure, the results for ${ }^{13} \mathrm{~N}-{ }^{13} \mathrm{C}$ are shown only for the four-cluster model. The two-cluster model predicts very similar values for $S_{p} / S_{n}$ for this mirror pair.

One can see that for the mirror pairs ${ }^{13} \mathrm{~N}-{ }^{13} \mathrm{C}$ and ${ }^{17} \mathrm{~F}$ ${ }^{17} \mathrm{O}$ with well developed single-particle structure, $S_{p} / S_{n}$ is very close to one. For most other cases the deviation of $S_{p} / S_{n}$ from one is no more than $4 \%$ for both the $N N$ potentials. The strongest deviation is obtained for the small $j=1 / 2$ components of the $\left\langle{ }^{8} \mathrm{~B} \mid{ }^{7} \mathrm{Be}\right\rangle$ and $\left\langle{ }^{8} \mathrm{Li} \mid{ }^{7} \mathrm{Li}\right\rangle$ overlap integrals and it reaches about $20 \%$ for $\mathrm{V} 2$ and $11 \%$ for MN potential, respectively. Such a sensitivity to the $N N$ potential for $j=1 / 2$ is explained by the different amounts of charge symmetry breaking required to reproduce the experimental separation energies in ${ }^{8} \mathrm{~B}$ and ${ }^{8} \mathrm{Li}$ with V2 and MN. Another strong deviation of $S_{p} / S_{n}$ from one occurs for the ${ }^{27} \mathrm{P}-{ }^{27} \mathrm{Mg}$ mirror pair where it reaches 8 to $9 \%$. This deviation arises from core excitation effects.

\subsection{Mirror symmetry in single-particle ANCs}

The overlap integrals $I_{l j}(r)$, divided by the square roots of their spectroscopic factors $S_{l j}$, are normalised functions of only one degree of freedom. They play the same role as single-particle wave functions generated by some effective local single-particle potential. These functions are characterised by the single-particle ANCs $b_{l j}=C_{l j} S_{l j}^{-1 / 2}$. Comparison between single-particle ANCs $b_{l j}$ for mirror nuclei may help to understand if mirror symmetry of the effective local single-particle potential wells is valid.

In this section we compare the ratio $\mathcal{R}_{b}=$ $C_{p}^{2} S_{n} /\left(C_{n}^{2} S_{p}\right)$ calculated in the microscopic cluster model with charge symmetry breaking and the ratio $\mathcal{R}_{\text {s.p. }}$. obtained using the same single-particle potential well which also reproduces experimental separation energies. If the charge symmetry of the local effective single-particle potentials is valid, then the ratio $\mathcal{R}_{b} / \mathcal{R}_{\text {s.p. }}$. should be equal to one. 


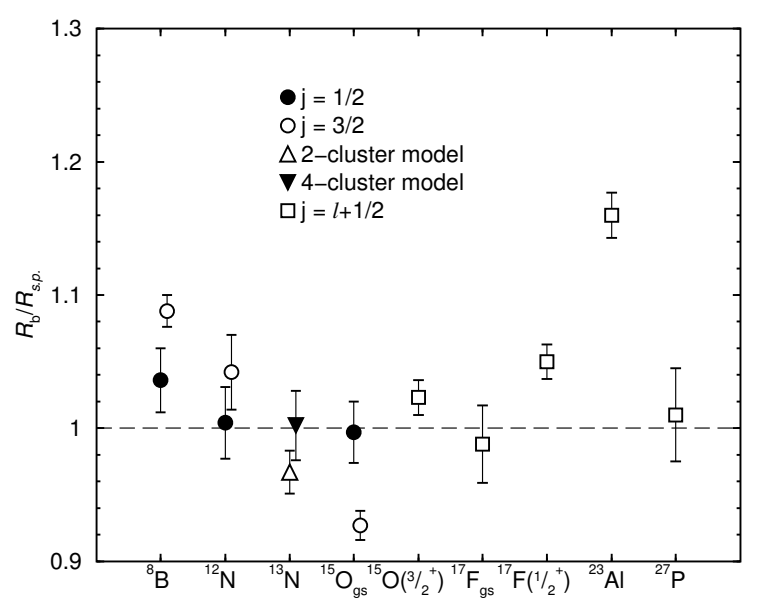

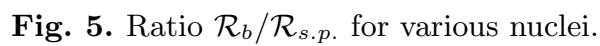

In fig. 5 we plot the ratio $\mathcal{R}_{b} / \mathcal{R}_{\text {s.p. }}$. The results of microscopic calculations for $\mathcal{R}_{b}$ with two different $N N$ potentials are averaged. The error bars in this figure are present due to this averaging and because of uncertainties in $\mathcal{R}_{\text {s.p. }}$ due to the residual dependence on nucleon-core potential.

For the mirror pairs of overlap integrals $\left\langle{ }^{8} \mathrm{~B} \mid{ }^{7} \mathrm{Be}\right\rangle-$ $\left\langle{ }^{8} \mathrm{Li} \mid{ }^{7} \mathrm{Li}\right\rangle,\left\langle{ }^{12} \mathrm{~N} \mid{ }^{11} \mathrm{C}\right\rangle-\left\langle{ }^{12} \mathrm{~B} \mid{ }^{11} \mathrm{~B}\right\rangle$, and $\left\langle{ }^{15} \mathrm{O} \mid{ }^{14} \mathrm{~N}\right\rangle-\left\langle{ }^{15} \mathrm{~N} \mid{ }^{14} \mathrm{~N}\right\rangle$ with $l=1$, where two angular momenta $j$ are possible, the ratio $\mathcal{R}_{b} / \mathcal{R}_{\text {s.p. }}$ is different in channels with $j=3 / 2$ and $j=1 / 2$. The deviation of $\mathcal{R}_{b} / \mathcal{R}_{\text {s.p. }}$ from one for these overlaps correlates with the size of their r.m.s. radii so that the larger is the r.m.s radius, the smaller are the deviations. For the $j=3 / 2$ overlaps $\left\langle{ }^{8} \mathrm{~B} \mid{ }^{7} \mathrm{Be}\right\rangle-\left\langle{ }^{8} \mathrm{Li} \mid{ }^{7} \mathrm{Li}\right\rangle$ and $\left\langle{ }^{12} \mathrm{~N} \mid{ }^{11} \mathrm{C}\right\rangle-\left\langle{ }^{12} \mathrm{~N} \mid{ }^{11} \mathrm{C}\right\rangle\left\langle r^{2}\right\rangle_{j=3 / 2}^{1 / 2}$ is larger than $\left\langle r^{2}\right\rangle_{j=1 / 2}^{1 / 2}[12]$

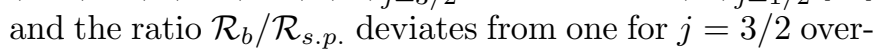
laps more than in the $j=1 / 2$ case. The situation for the $\left\langle{ }^{15} \mathrm{O} \mid{ }^{14} \mathrm{~N}\right\rangle-\left\langle{ }^{15} \mathrm{~N} \mid{ }^{14} \mathrm{~N}\right\rangle$ mirror overlaps is opposite, the r.m.s. radius for $j=1 / 2$ is smaller than that for $j=3 / 2$ and $\mathcal{R}_{b}$ deviates from $\mathcal{R}_{\text {s.p. }}$ more for the latter case.

For the $\left\langle{ }^{13} \mathrm{~N} \mid{ }^{12} \mathrm{C}\right\rangle-\left\langle{ }^{13} \mathrm{C} \mid{ }^{12} \mathrm{C}\right\rangle$ pair of mirror overlaps, we have performed the calculations both in four- and twocluster models. In the first case, the mirror symmetry of local effective potential wells is valid. In the second case, the mirror local effective potentials are not the same due to large charge symmetry breaking required to reproduce the experimental separation energies.

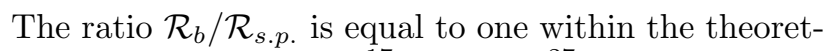
ical uncertainties only for ${ }^{17} \mathrm{~F}_{\text {g.s. }}$. and ${ }^{27} \mathrm{P}$. It is interesting that significant deviation between $\mathcal{R}_{b}$ and $\mathcal{R}_{\text {s.p. }}$. can be seen for the single-particle nuclear state ${ }^{17} \mathrm{~F}\left(\frac{1}{2}^{+}\right)$, in which the mirror symmetry of the mean field is intuitively expected. We believe that the reason for such a deviation is the fact that in ${ }^{17} \mathrm{O}\left(\frac{1}{2}^{+}\right)$the valence 1 s neutron penetrates inside the core more easily than the mirror proton thus more strongly disturbing the mean field. Also a very strong deviation occurs for ${ }^{23} \mathrm{Al}$ which should originate from the strong deformation of the ${ }^{22} \mathrm{Mg}$ core.

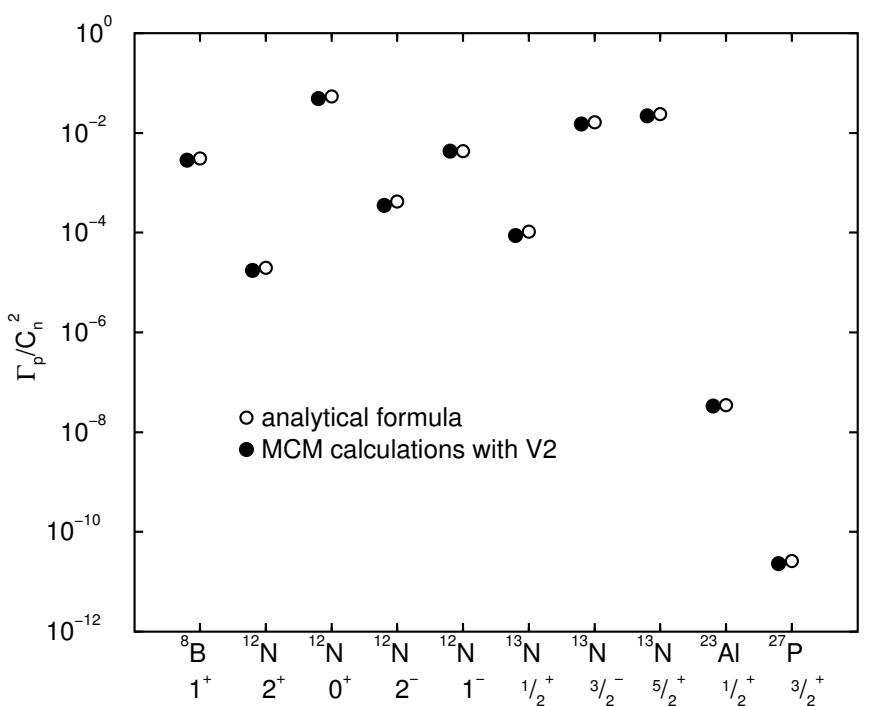

Fig. 6. Ratio of the proton width to the ANC squared of the mirror neutron calculated (given in the units of $\hbar c$ ) with exactly the same $N N$ interactions in mirror nuclei as compared to the predictions of the analytical formula (11).

\section{Bound-unbound mirror pairs}

In this section we consider excited mirror states, that lie above proton emission thresholds on the proton-rich side and are bound on the neutron-rich side, for the same $0 p$ and $s d$ shell mirror pairs as in the previous section.

First of all we perform the microscopic cluster model calculations with exactly the same $N N$ potentials for each nucleus of a mirror pair, thus imposing charge symmetry of the $N N$ interactions. The adjustable parameters of the $N N$ potentials are chosen to reproduce the experimental energies of proton resonances. We calculate the widths $\Gamma_{p}$ for these resonances, the ANCs squared $C_{n}^{2}$ for mirror neutrons and plot their ratio $\mathcal{R}_{\Gamma}^{M C M}$ in fig. 6 for the $\mathrm{V} 2$ potential. We compare this ratio to the prediction $\mathcal{R}_{0}^{\text {res }}$ of the analytical formula (11). As seen in fig. 6, the analytical formula describes very well the general trend in the $\mathcal{R}_{\Gamma}^{M C M}$ behaviour. The same is true for the MN potential.

To see the differences between $\mathcal{R}_{\Gamma}^{M C M}$ and $\mathcal{R}_{0}^{\text {res }}$ we have plotted in fig. 7 the ratio $\mathcal{R}_{0}^{\text {res }} / \mathcal{R}_{\Gamma}^{M C M}$. We calculated this ratio both in the single-channel (no core excitations) and the multi-channel (including several core excitations) cluster model for two different $N N$ potentials, V2 and MN. The results are plotted in fig. 7. One can see from this figure that for $0 p$ shell nuclei the results obtained with and without taking core excitations into account differ by not more than $6 \%$, except for ${ }^{12} \mathrm{~N}\left(0^{+}\right)$, where this difference is about $10 \%$. Core excitations become more important for nuclei in the middle of the $s d$ shell. For ${ }^{23} \mathrm{Ne}^{23} \mathrm{Al}$, their influence on $\mathcal{R}_{\Gamma}^{M C M}$ is about $12-16 \%$. A similar effect is seen for ${ }^{27} \mathrm{Mg}\left(\frac{3}{2}^{+}\right){ }^{27} \mathrm{P}\left(\frac{3}{2}^{+}\right)$in the calculations with the V2 potential but for MN this influence is much stronger, about $45 \%$. This happens because with the MN potential the $d$-wave ${ }^{26} \mathrm{Si}\left(0^{+}\right)+\mathrm{p}$ configuration in ${ }^{27} \mathrm{P}\left(\frac{3}{2}^{+}\right)$becomes three times weaker than 

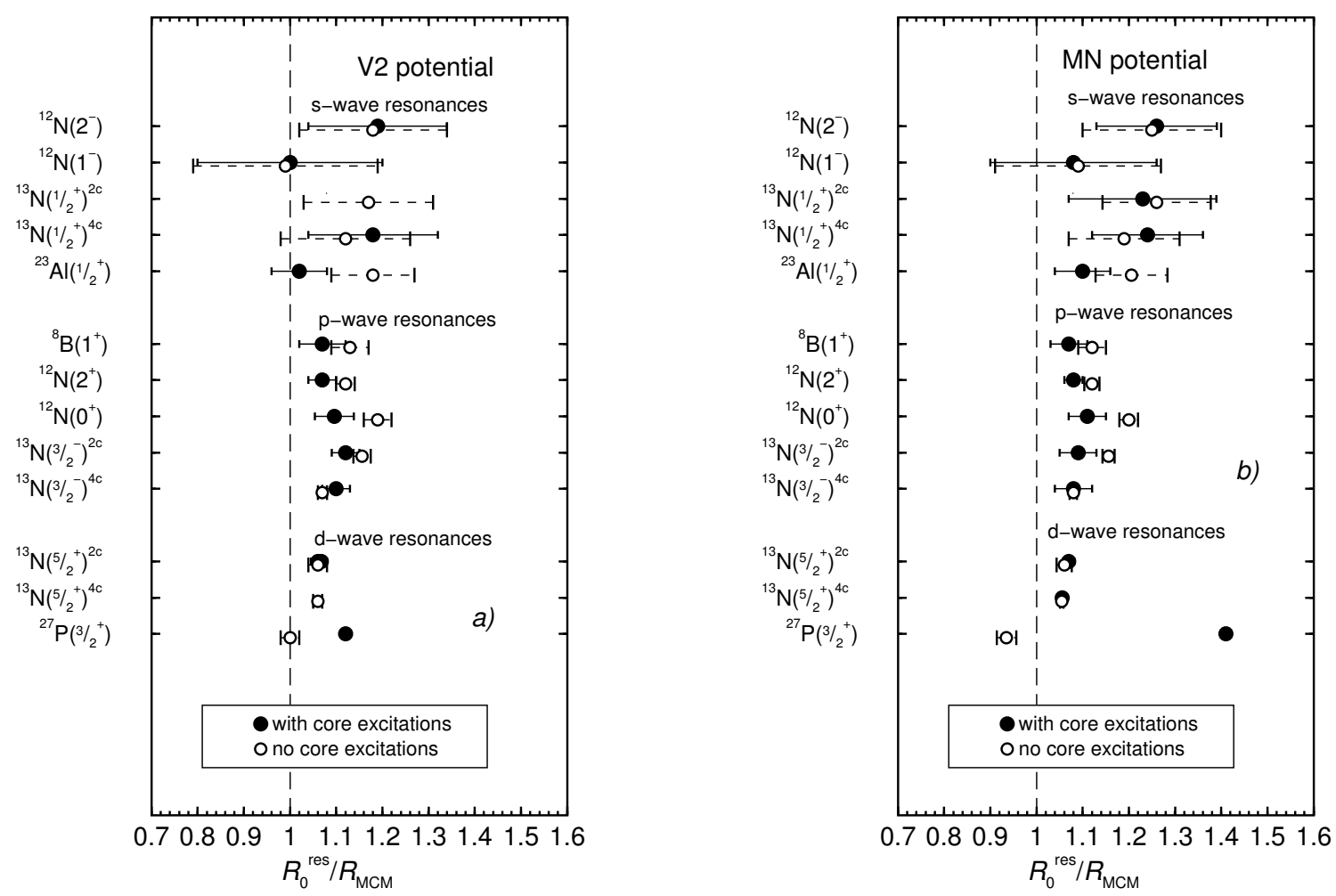

Fig. 7. Ratio between the predictions $\mathcal{R}_{0}^{\text {res }}$ of the analytical formula (11) and those from the microscopic calculations $\mathcal{R}_{\Gamma}^{M C M}$ for the V2 $(a)$ and $\mathrm{MN}(b)$ potentials with and without taking core excitations into account. Charge symmetry of the $N N$ interactions is assumed. Both four-cluster $(4 c)$ and two-cluster $(2 c)$ calculations for ${ }^{13} \mathrm{~N}$ are shown.

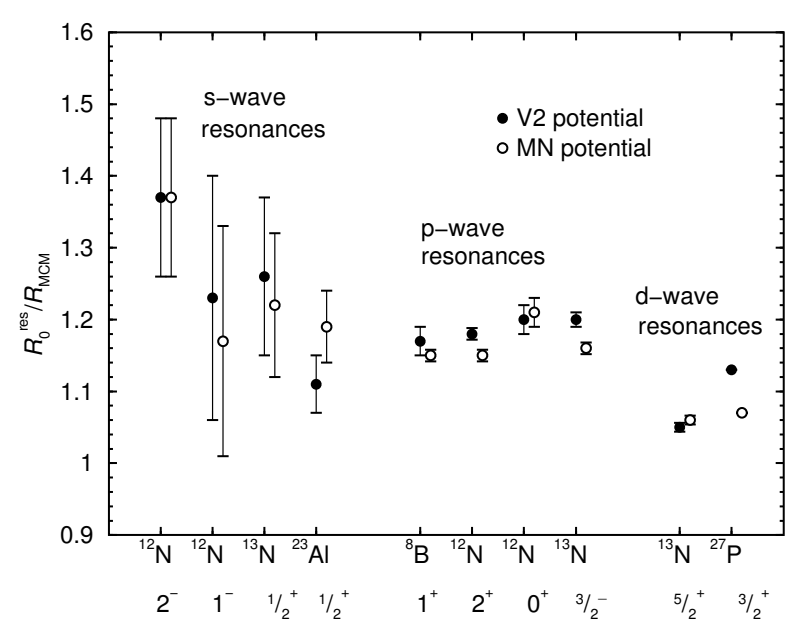

Fig. 8. Ratio between the predictions $\mathcal{R}_{0}^{\text {res }}$ of the analytical formula (11) and the microscopic calculations $\mathcal{R}_{\Gamma}^{M C M}$ for the $\mathrm{V} 2$ and MN potentials. The $N N$ potentials are slightly different in mirror nuclei. Core excitations are included. For ${ }^{13} \mathrm{~N}$, the results of the four-cluster calculations are shown.

the $s$-wave ${ }^{26} \mathrm{Si}\left(2^{+}\right)+\mathrm{p}$ configuration. In weak configurations the effects of charge symmetry breaking due to the Coulomb interaction are more noticeable. In the particular case of ${ }^{27} \mathrm{Mg}\left(\frac{3}{2}^{+}\right)-{ }^{27} \mathrm{P}\left(\frac{3}{2}^{+}\right)$, the mirror symmetry breaking in spectroscopic factors, obtained with MN potential, is about $33 \%$, while with V2 the $d$-wave ${ }^{26} \mathrm{Si}\left(0^{+}\right)+$p config- uration dominates and the mirror symmetry breaking for spectroscopic factor of this configuration is only $4 \%$. For other nuclei, the effect of different choices of the $N N$ interaction is about 6 to $8 \%$. The average deviation of $\mathcal{R}_{0}^{\text {res }}$ from $\mathcal{R}_{M C M}$ is about $10-12 \%$.

Next, we perform multi-channel calculations in which the experimental energies of proton resonance and neutron separation energies are reproduced. This requires some charge symmetry breaking in the $N N$ interactions used. The results of such calculations are presented in fig. 8 . The differences in the calculations with V2 and MN potentials do not exceed $8 \%$ for all the cases considered here. The largest difference between $\mathcal{R}_{0}^{\text {res }}$ and $\mathcal{R}_{\Gamma}^{M C M}$ occurs, as expected, for the $s$-wave resonances ${ }^{12} \mathrm{~N}\left(2^{-}\right),{ }^{12} \mathrm{~N}\left(1^{-}\right)$ and ${ }^{13} \mathrm{~N}\left(\frac{1}{2}^{-}\right)$, the widths of which are not small. For the narrow $s$-wave resonance ${ }^{23} \mathrm{Al}, \mathcal{R}_{0}^{\text {res }}$ deviates from $\mathcal{R}_{\Gamma}^{M C M}$ by about $14 \%$. Similar deviations, of $16-18 \%$, occur for all the $p$-wave resonances. For the $d$-wave resonances ${ }^{13} \mathrm{~N}\left(\frac{5}{2}^{+}\right)$ and ${ }^{27} \mathrm{P}\left(\frac{3}{2}^{+}\right)$this deviation is noticeably smaller, $6 \%$ and $10 \%$ respectively.

\section{Discussion and conclusions}

In this paper we have performed calculations of ANCs for mirror one-body overlap integrals within a multi-channel microscopic cluster model. These calculations provide an improved understanding of mirror symmetry in ANCs 
because the model used takes into account the differences in the internal structure of mirror nuclei due to the Coulomb interaction and the effects that arise due to core excitations, which were ignored in the derivation of the analytical formula (9). Comparison of the results obtained within the microscopic model with the predictions of eq. (9) has confirmed the general trend in the behaviour of ratio of mirror ANCs, given by this formula. This trend is determined only by the separation energies of mirror proton and neutron and the charges of the cores. The difference between the microscopic calculations and the analytical formula is usually less than $7 \%$. However, it may reach, 10 to $12 \%$ for the very weakly bound $1 s$ nuclei and for nuclei with strongly excited cores.

The microscopic calculations of the ratio of mirror ANCs are also very close to those of the single-particle model, where mirror symmetry in the single-particle potential wells and in the spectroscopic factors is assumed. The difference between such calculations is typically less that $7 \%$ except for the $s d$ shell nuclei ${ }^{23} \mathrm{Al}$ and ${ }^{27} \mathrm{P}$ with strongly deformed cores. The average of the single-particle estimate and the prediction of the analytical model is in agreement with the microscopic calculations to within $6 \%$, except for ${ }^{23} \mathrm{Al}$. Therefore, this average value can be used to predict unknown ANCs from known mirror ones when the microscopic calculations are not available. As far as the mirror symmetry of the single-particle model is concerned, it is not always justified. Our microscopic calculations have shown that spectroscopic factors in mirror states can differ by up to $9 \%$ and that mirror symmetry in proton and neutron potential wells is not always present, even for nuclear states with well-pronounced single-particle structure.

Mirror symmetry of ANCs can be used to predict cross sections of proton capture at stellar energies using mirror neutron ANCs. For example, the astrophysical $S$-factor of the ${ }^{7} \mathrm{Be}(\mathrm{p}, \gamma){ }^{8} \mathrm{~B}$ reaction can be calculated using the ANCs for the overlap integral $\left\langle{ }^{8} \mathrm{Li} \mid{ }^{7} \mathrm{Li}\right\rangle$. The latter has been measured recently in [13]. Using this experimental value and the predictions for the ratio of the ${ }^{8} \mathrm{~B}$ proton $\mathrm{ANC}$ to the ${ }^{8} \mathrm{Li}$ neutron ANC from the microscopic cluster calculations, we get $S_{17}(0)=17.8 \pm 1.7 \mathrm{eV} \cdot \mathrm{b}$ for $\mathrm{V} 2$ and $18.2 \pm 1.8 \mathrm{eV} \cdot \mathrm{b}$ for the MN. These results agree well with most measurements based on indirect methods.

The microscopic calculations for bound-unbound mirror states have confirmed that the main trend in the behaviour of the ratio between the proton width and the mirror neutron ANC squared is well reproduced by the analytical formula (11). The difference between the predictions of this formula and the exact microscopic calculations is less than $20 \%$ for narrow proton resonances.

The mirror symmetry between the proton width and the mirror neutron ANC can be used to predict unknown widths of very narrow resonances.
It can also be used as a test of the accuracy for experimentally measured ANCs. For example, for the neutron $\mathrm{ANC}$ of ${ }^{8} \mathrm{Li}\left(1^{+}\right)$measured in [13] and the proton width of ${ }^{8} \mathrm{~B}\left(1^{+}\right)$from [14] we get $\mathcal{R}_{\text {exp }}=(2.29 \pm 0.28) \times$ $10^{-3} \hbar c$. This is significantly larger than the predictions $\mathcal{R}_{\Gamma}^{M C M}=(1.73 \pm 0.03) \times 10^{-3} \hbar c$ of the microscopic model. The proton width of ${ }^{8} \mathrm{~B}\left(1^{+}\right)$has recently been remeasured in ${ }^{7} \mathrm{Be}+\mathrm{p}$ scattering [15], which leads to $\mathcal{R}_{\text {exp }}=(1.92 \pm 0.23) \times 10^{-3} \hbar c$. The remaining difference between $\mathcal{R}_{\text {exp }}$ and $\mathcal{R}_{\Gamma}^{M C M}$ requires the verification of the accuracy of the determination of the ${ }^{8} \mathrm{Li}\left(1^{+}\right)$ANC.

This work has been supported by the UK EPSRC via grant GR/T28577.

\section{References}

1. H.M. Xu, C.A. Gagliardi, R.E. Tribble, A.M. Mukhamedzhanov, N.K. Timofeyuk, Phys. Rev. Lett. 73, 2027 (1994).

2. N.K. Timofeyuk, R.C. Johnson, A.M. Mukhamedzhanov, Phys. Rev. Lett. 91, 232501 (2003).

3. C. Iliadis, L. Buchmann, P.M. Endt, H. Herndl, M. Wiescher, Phys. Rev. C 53, 475 (1996).

4. L.D. Blokhintsev, I. Borbely, E.I. Dolinskii, Sov. J. Part. Nucl. 8, 485 (1977).

5. A.M. Mukhamedzhanov, N.K. Timofeyuk, Sov. J. Nucl. Phys. 51, 431 (1990), (Yad. Fiz. 51679 (1990)).

6. N.K. Timofeyuk, Nucl. Phys. A 632, 38 (1998).

7. A.M. Mukhamedzhanov, R.E. Tribble, Phys. Rev. C 59, 3418 (1999).

8. P. Descouvemont, M. Vincke, Phys. Rev. A 42, 3835 (1990).

9. A.B. Volkov, Nucl. Phys. 74, 33 (1965).

10. D.R. Thompson, M. LeMere, Y.C. Tang, Nucl. Phys. A 286, 53 (1977).

11. D. Baye, N. Pecher, Bull. Sc. Acad. Roy. Belg. 67, 835 (1981).

12. N.K. Timofeyuk, P. Descouvemont, Phys. Rev. C 71, 064305 (2005).

13. L. Trache, A. Azhari, F. Carstoiu, H.L. Clark, C.A. Gagliardi, Y.-W. Lui, A.M. Mukhamedzhanov, X. Tang, N. Timofeyuk, R.E. Tribble, Phys. Rev. C 67, 062801(R) (2003).

14. F. Ajzenberg-Selove, Nucl. Phys. A 490, 1 (1988).

15. C. Angulo, M. Azzouz, P. Descouvemont, G. Tabacaru, D. Baye, M. Cogneau, M. Couder, T. Davinson, A. Di Pietro, P. Figuera, M. Gaelens, P. Leleux, M. Loiselet, A. Ninane, F. de Oliveira Santos, R.G. Pizzone, G. Ryckewaert, N. de Sereville, F. Vanderbist, Nucl. Phys. A 716, 211 (2003). 